\title{
Electrochemical Deposition of Zinc Oxide on the Surface of Composite Membrane Polysulfone-Graphene-Polystyrene in the Presence of Water Soluble Polymers
}

\author{
Alexandra Mocanu, ${ }^{1}$ Edina Rusen, ${ }^{2}$ Aurel Diacon, ${ }^{2}$ Celina Damian, ${ }^{2}$ \\ Adrian Dinescu, ${ }^{3}$ and Mirela Suchea ${ }^{4,5}$ \\ ${ }^{1}$ Department of Chemical and Biochemical Engineering, University Politehnica of Bucharest, 1-7 Gh. Polizu Street, \\ 011061 Bucharest, Romania \\ ${ }^{2}$ Department of Bioresources and Polymer Science, University Politehnica of Bucharest, 1-7 Gh. Polizu Street, \\ 011061 Bucharest, Romania \\ ${ }^{3}$ National Institute for Research and Development in Microtechnologies (IMT-Bucharest), 126 A, Erou Iancu Nicolae Street, \\ P.O. Box 38-160, 023573 Bucharest, Romania \\ ${ }^{4}$ Center of Materials Technology and Photonics, School of Engineering, Technological Educational Institute of Crete, \\ 71004 Heraklion, Greece \\ ${ }^{5}$ Chemistry Faculty, “Alexandru Ioan Cuza” University of Iaşi, 11 Carol I Blvd, 700506 Iaşi, Romania
}

Correspondence should be addressed to Edina Rusen; edina_rusen@yahoo.com and Aurel Diacon; aurel_diacon@yahoo.com

Received 27 November 2016; Revised 14 February 2017; Accepted 26 February 2017; Published 9 March 2017

Academic Editor: Michele Zappalorto

Copyright ( 2017 Alexandra Mocanu et al. This is an open access article distributed under the Creative Commons Attribution License, which permits unrestricted use, distribution, and reproduction in any medium, provided the original work is properly cited.

\begin{abstract}
The aim of this study consisted in the development of an alternative synthesis procedure for hybrid ultrafiltration membranes for water purification. The membranes were obtained by wet-phase inversion method based on aliquots of polysulfone (PSF) and graphene nanoplatelets modified with poly(styrene) (G-PST). The hybrid materials were modified by electrochemical deposition of zinc oxide $(\mathrm{ZnO})$ on one side of the membranes in the presence of water soluble polymers. Raman, XPS, and TGA analyses were used to characterize the chemical and thermal characteristics of the PST-G. SEM analysis showed the formation of asymmetric porous configuration in all cases and the generation of $\mathrm{ZnO}$ with different shapes/structures on the bottom surface of the membrane or inside the porous channels. EDS analysis confirmed the formation of $\mathrm{ZnO}$.
\end{abstract}

\section{Introduction}

Water purification continues to be considered a major problem of the current century although tremendous efforts were made at industrial level to improve membrane technology.

Regardless of their classification microfiltration (MF, pores $0.1-1 \mu \mathrm{m}$ ), ultrafiltration (UF, $0.01-0.1 \mu \mathrm{m}$ ), nanofiltration (NF, $1-10 \mathrm{~nm}$ ), and reverse osmosis (RO, nonporous) membranes designed for water desalination or wastewater treatment have to meet several requirements regarding high selectivity/retention grade to contaminants, high mechanical resistance, and low-cost manufacturing process [1].
Although at industrial level polymer membranes such as poly(vinylidene fluoride) (PVDF), polysulfone (PSF), and poly(ethersulfone) (PES) are the most widely used also for their thermal and chemical resistance $[2,3]$, the poor resistance to fouling limits their performance.

For this reason, research studies were driven to increasing the hydrophilicity of the polymer membranes by physical blending with more hydrophilic polymers or inorganic compounds, by chemical and/or surface modification [3-6].

The filtration membranes were obtained using the classical immersion phase inversion method starting from aliquots of polymer solutions with previously synthesized inorganic particles like zinc oxide $(\mathrm{ZnO})$, titanium dioxide $\left(\mathrm{TiO}_{2}\right)$, 
alumina $\left(\mathrm{Al}_{2} \mathrm{O}_{3}\right)$, silica $\left(\mathrm{SiO}_{2}\right)$, silver, or carbon [2, 4, 7-9]. This strategy not only determined the modification of pores morphology, but also had an important effect on higher water fluxes and better antifouling properties due to the hydrophilic increased nature of the resulting membranes.

Polymer-inorganic nanocomposites have attracted significant scientific and technological interest. The incorporation of inorganic nanoparticles into the polymer matrix can provide high-performance novel materials that find applications in many industrial fields. As a result of the development in nanotechnology, inorganic nanostructured materials have been designed and fabricated with important cooperative physical phenomena such as superparamagnetism, sizedependent band-gap, ferromagnetism, and electron and phonon transport $[10,11]$.

Carbon nanomaterials possess some unusual size-dependent properties (e.g., morphological, electrical, optical, and mechanical) useful in enhancing energy-conversion, storage performance, and mechanical properties [12, 13]. Specifically, considerable efforts have been made to utilize the unique properties of fullerenes, carbon nanotubes, and graphene as energy materials, and tremendous progress has been achieved in developing carbon nanomaterials for highperformance energy-conversion and storage devices.

Furthermore, incorporation of pristine carbon nanotubes (CNTs) or graphene oxide (GO) at certain concentrations into the polymer matrix proved to be an advantageous alternative to realize filtration membranes with improved hydrophilicity, thermal stability, and water flux permeability without damaging the mechanical properties of the hybrid membranes $[5,14-16]$.

For complementary purposes composite filtration membranes were obtained using both inorganic nanoparticles and CNTs or GO in order to significantly increase the fouling resistance and in some cases to obtain additional photocatalytic or antimicrobial properties $[17,18]$.

In our study graphene nanosheets were modified with polystyrene in order to favour a better dispersion of the sheets into the polysulfone matrix. The hybrid polysulfonepoly(styrene)-graphene (PSF-G-PST) membranes were synthesized by casting the solution of PSF and G-PST dissolved in N-methyl-pyrrolidone (NMP) on glass plates followed by coagulation in water bath. The electroconductive properties of the synthesized membranes were exploited for the electrochemical deposition of $\mathrm{ZnO}$. In order to control the $\mathrm{ZnO}$ morphology, the electrochemical procedure was applied in the presence of water soluble compounds in order to form $\mathrm{ZnO}$ nanoparticles on one side of the composite membrane. The PSF membrane is hydrophobic. $\mathrm{ZnO}$ was generated in the presence of hydrophile polymers. Thus, $\mathrm{ZnO}$ was deposited on the surface of the PSF membrane in order to increase the membrane antifouling properties.

The chemical modification and thermal behaviour of modified graphenes were investigated by Raman spectroscopy, X-ray photon spectroscopy (XPS), and thermogravimetric analysis (TGA). Scanning electron microscopy (SEM) was employed to investigate the pores structures and $\mathrm{ZnO}$ nanoparticles modifications depending on the water soluble compounds. Energy dispersive spectroscopy (EDS) evidenced the formation of $\mathrm{ZnO}$ on the surface of the hybrid membranes.

\section{Materials and Methods}

2.1. Materials. Graphene nanoplatelets $(5 \mu \mathrm{m}$ wide, average $5 \mathrm{~nm}$ thickness, bulk density of $0.03-0.1 \mathrm{~g} / \mathrm{cc}$, carbon content $>99.5 \mathrm{wt} \%$, oxygen content $<1 \%$, and residual acid content $<0.5 \mathrm{wt} \%$ ) (G) (Emfutur Technologies Ltd., Spain) were used as received. Styrene (ST) (Aldrich) has been purified through distillation under vacuum. Potassium chloride (KCl) (Aldrich) has been recrystallized from an ethanol/water mixture and then vacuum dried until constant mass. Polysulfone beads (average Mn 22,000) (PSF) (Aldrich), 2-Hydroxyethyl cellulose (average $\mathrm{Mw}=250,000$ ) (HEC) (Aldrich), Poly(vinyl alcohol) (average $\mathrm{Mw}=85,000-$ 124,000, 87-89\% hydrolysed) (PVA) (Aldrich), Poly(acrylic acid) (average $\mathrm{Mw}=1800)(\mathrm{APA})($ Aldrich), methanol $(\mathrm{M})$ (Merck), dimethylformamide (DMF) (Sigma), 1-N-methyl-2pyrrolidinone (NMP) (Aldrich), and zinc nitrate hexahydrate (purum p.a., crystallized, $\geq 99.0 \%)\left(\mathrm{Zn}\left(\mathrm{NO}_{3}\right)_{2} \cdot 6 \mathrm{H}_{2} \mathrm{O}\right.$ ) (Honeywell) have been used with no previous purification.

\subsection{Methods}

2.2.1. Synthesis of Graphene-Polystyrene (G-PST) Composite. A black dispersion of $150 \mathrm{mg}$ of $\mathrm{G}$ in $8 \mathrm{~mL}$ of ST was added to $10 \mathrm{~mL}$ of DMF followed by nitrogen purging for $20 \mathrm{~min}$. The reaction was initiated with $100 \mathrm{mg}$ of PL (lauroyl peroxide) and kept under continuous stirring overnight at $80^{\circ} \mathrm{C}$. The resulting product (G-PST) was purified by precipitation in M. Finally, the gray-black powder was vacuum dried until constant mass.

2.2.2. Synthesis of Polysulfone-Graphene-Poly(styrene) (PSFG-PST) Composite Membranes. The composite membranes were obtained by the wet-phase inversion method [19] in which a $15 \%$ PSF and $0,2 \%$ G-PST (wt.\% by PSF content) were dissolved in NMP and kept at room temperature under continuous stirring for $12 \mathrm{~h}$. The casting of the membranes was performed on glass plates immediately followed by coagulation in deionized water bath. The membranes were kept in the water bath until complete detachment of the hybrid membranes and moved afterwards for another $12 \mathrm{~h}$ in fresh deionized water in order to remove the entire solvent and to favour the complete formation of the pores. The PSFG-PST membranes were dried for the next $12 \mathrm{~h}$ at room temperature.

\subsubsection{Electrochemical Deposition of $\mathrm{ZnO}$ on the Surface of} the PSF-G-PST Membranes. The $\mathrm{ZnO}$ nanostructures were generated on one side of the hybrid membranes using a modified electrochemical method described elsewhere [20]. Hybrid membranes were cut in small pieces $(1 \times 1 \mathrm{~cm})$ and used as cathodes, while a $\mathrm{Pt}$ wire was fixed as the anode. Solutions containing $0.5 \mathrm{~g} \mathrm{Zn}\left(\mathrm{NO}_{3}\right)_{2} \cdot 6 \mathrm{H}_{2} \mathrm{O}, 0.5 \mathrm{~g} \mathrm{KCl}$ and $20 \mathrm{~mL}$ distilled water for blank experiment were continuously stirred at room temperature for $1 \mathrm{~h}$, at a potential value of $-1.5 \mathrm{~V}$. In the case of $\mathrm{ZnO}$ generation in presence of water 
TABLE 1: Electrochemical deposition conditions for $\mathrm{ZnO}$ generation on the composite membrane.

\begin{tabular}{lccc}
\hline Sample code & Polymer & $\begin{array}{c}\text { Concentration of polymer in solution } \\
(\% \text { wt. })\end{array}$ & $\begin{array}{c}\text { Deposition time } \\
(\text { h) }\end{array}$ \\
\hline PSF-G-PST-PVA 2.5 & PVA & 2.5 & 1 \\
PSF-G-PST-PVA 10 & PVA & 10 & 1 \\
PSF-G-PST-PAA 2.5 & PAA & 2.5 & 1 \\
PSF-G-PST-HEC 2.5 & HEC & 2.5 & 1 \\
\hline
\end{tabular}

soluble polymers different concentration and polymers were employed according to Table 1 . At the end of all experiments, the composite membranes were removed from the electrochemical bath and washed several times with distilled water, followed by drying at $60^{\circ} \mathrm{C}$ until constant mass.

\section{Characterization}

Graphene chemical functionalization was studied by Raman and XPS spectroscopy. The Raman spectra have been registered on a DXR Raman Microscope from Thermo Scientific with a $633 \mathrm{~nm}$ laser. The laser beam has been focused with a 10x objective. The XPS (X-ray photoelectron microscopy) analysis has been performed on a K-Alpha instrument from Thermo Scientific, using a monochromated Al K $\alpha$ source $(1486.6 \mathrm{eV})$, at a low pressure of $2 \times 10^{-9} \mathrm{mbar}$. Charging effects have been compensated by a flood gun and binding energy has been calibrated by placing the Cls peak at $285 \mathrm{eV}$ as internal standard.

The thermal properties of the material were ascertained using thermogravimetric analysis (TGA). The TGA analysis has been performed on Q500 TA Instruments equipment, under oxygen atmosphere, using a heating rate of $20^{\circ} \mathrm{C} / \mathrm{min}$ from room temperature to $900^{\circ} \mathrm{C}$.

The morphologies of the membranes have been investigated by SEM (scanning electron microscopy) using FEGSEM-Nova NanoSEM 630 (FEI). The presence of $\mathrm{ZnO}$ nanostructures on the surface of the hybrid materials was confirmed by energy dispersive spectroscopy (EDS).

\section{Results and Discussions}

In this research study, the porous membranes were designed using an alternative synthesis procedure in order to meet various requirements necessary for membranes used in wastewater treatment applications. For this reason, the materials were modified to ensure good mechanical and thermal resistance and hydrophilic and antifouling properties simultaneously.

Although high amounts of graphene or graphene oxide can decrease mechanical properties of hybrid membranes, these fillers have been used in polymer membranes synthesis in order to increase thermal, hydrophilic, antifouling, and antibacterial properties of different polymer membranes. Due to its poor solubility in common organic solvents, graphene has been chemically modified in order to enhance the compatibility with other materials [21].

In our study, to favour a better dispersion of graphene sheets in polysulfone matrix, the polymer composite G-PST

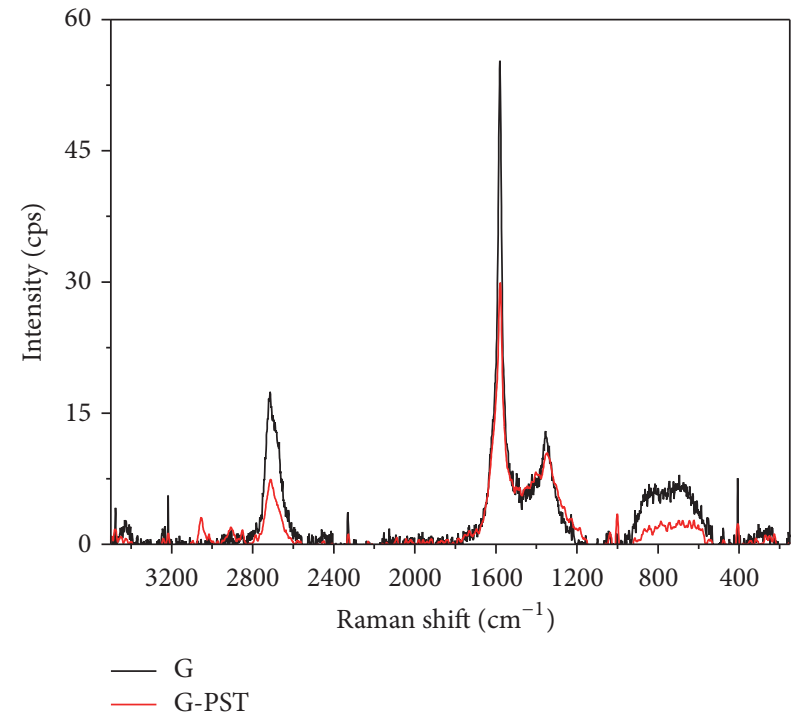

FIGURE 1: Raman analysis of G (black line) and modified G-PST (red line).

was obtained based on a solution polymerization reaction of styrene.

Raman and XPS analyses were performed in order to put into evidence the chemical modification of graphene with polystyrene, while TGA analysis was employed to establish the thermal properties of the G-PST composite.

The Raman spectrum (Figure 1, black line) confirmed the characteristic signals of graphene: (1) the primary in-plane vibrational mode, the $\mathrm{G}$ band $\left(1580 \mathrm{~cm}^{-1}\right)$, (2) the secondary in-plane vibrational mode, the $\mathrm{D}$ band due to defects presence, and (3) the second-order overtone of different in-plane vibration, namely, the $2 \mathrm{D}$ band $\left(2714 \mathrm{~cm}^{-1}\right)$. In our case, the peak for $\mathrm{G}$ band is slightly shifted and the shape of $2 \mathrm{D}$ band is not sharp comparing to single-layered graphenes, which proves a multilayered structure of the nanoplatelets according to Wang et al. [22]. For G-PST composite the Raman signals of graphene have lower intensities confirming the chemical modification (Figure 1, red line). The successful chemical functionalization of $\mathrm{G}$ is confirmed by the modification of the ratio $I_{\mathrm{G}}\left(1580 \mathrm{~cm}^{-1}\right) / I_{\mathrm{D}}\left(1350 \mathrm{~cm}^{-1}\right)$ [23] from 4.35 to 2.85 after PST grafting, thus confirming the increased number of defects in the graphene structure.

The XPS analysis from Figure 2(a) shows the high resolution scan for C1s species of the graphene that were deconvoluted into five secondary peaks: the first one at $284.8 \mathrm{eV}$ is 


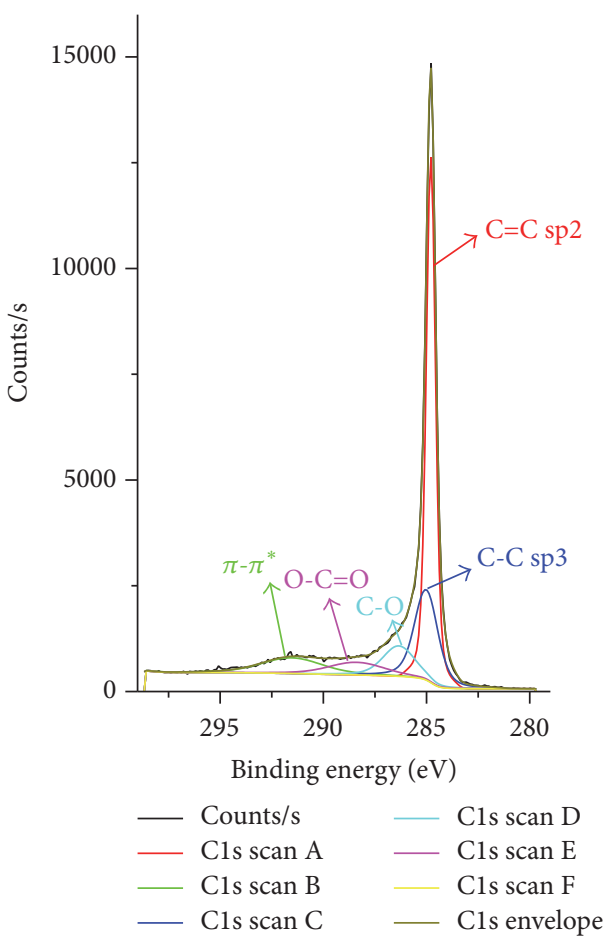

(a) Pristine G

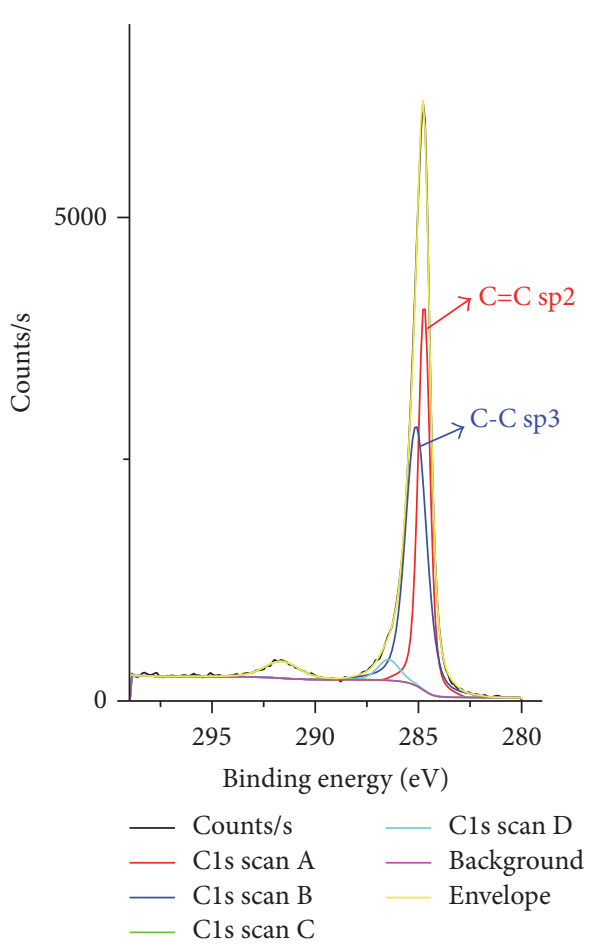

(b) G-PST

FIGURE 2: XPS analysis, C1s deconvolution spectra for G (a) and G-PST (b).

assigned to $\mathrm{sp}^{2}$ carbon atoms involved in $\mathrm{C}=\mathrm{C}$ type bonds, the second peak at $285.0 \mathrm{eV}$ is attributed to the presence of $\mathrm{sp}^{3}$ type carbon species which are defects in the structure of graphene lattice, the third peak from $286.3 \mathrm{eV}$ corresponds to the $\mathrm{C}-\mathrm{O}$ species, while the $288.4 \mathrm{eV}$ peak is known as the signal of $\mathrm{O}-\mathrm{C}=\mathrm{O}$ bonds. There is the fifth peak from $291.5 \mathrm{eV}$ which arises from $\pi-\pi^{*}$ electronic interactions of the graphene aromatic structure $[24,25]$. Meanwhile, oxygen reduction was also clearly observed during the functionalization process; thus, for G-PST sample (Figure 2(b)), the deconvoluted area of $\mathrm{C}-\mathrm{O}$ groups decreased clearly while the signal of $\mathrm{O}-\mathrm{C}=\mathrm{O}$ is completely lost; also it can be noticed that the amount of $\mathrm{C}-\mathrm{C} \mathrm{sp}{ }^{3}$ species at $285 \mathrm{eV}$ increased, indicating that the functionalization of $\mathrm{G}$ is mainly achieved via reaction with oxygenous groups, to form PST chains bonded covalently to $G$ surface $[26,27]$. The attack of oxygen functional groups might stem from the fact that the C-O binding energy $(351 \mathrm{~kJ} / \mathrm{mol})$ is lower than the $\mathrm{C}-\mathrm{C}$ binding energy $(368 \mathrm{~kJ} / \mathrm{mol})[28]$.

As exhibited in Figure 3, there is a slight weight loss (approximately $10 \%$ ) in the case of $\mathrm{G}$ nanoplatelets at $500^{\circ} \mathrm{C}$ that can be attributed to the labile oxygen groups proving a high carbon content. In the case of G-PST nanocomposite there is also a slight weight loss (around 5\%) at temperature below $100^{\circ} \mathrm{C}$ due to the removal of adsorbed water on the surface of the composite. The thermal decomposition temperature of G-PST is registered at almost $380^{\circ} \mathrm{C}$, while the complete degradation appears at $450^{\circ} \mathrm{C}$ due to the pyrolysis of PST chains. The presence of $\mathrm{G}$ nanoplatelets improved the thermal properties of the composite considering that the

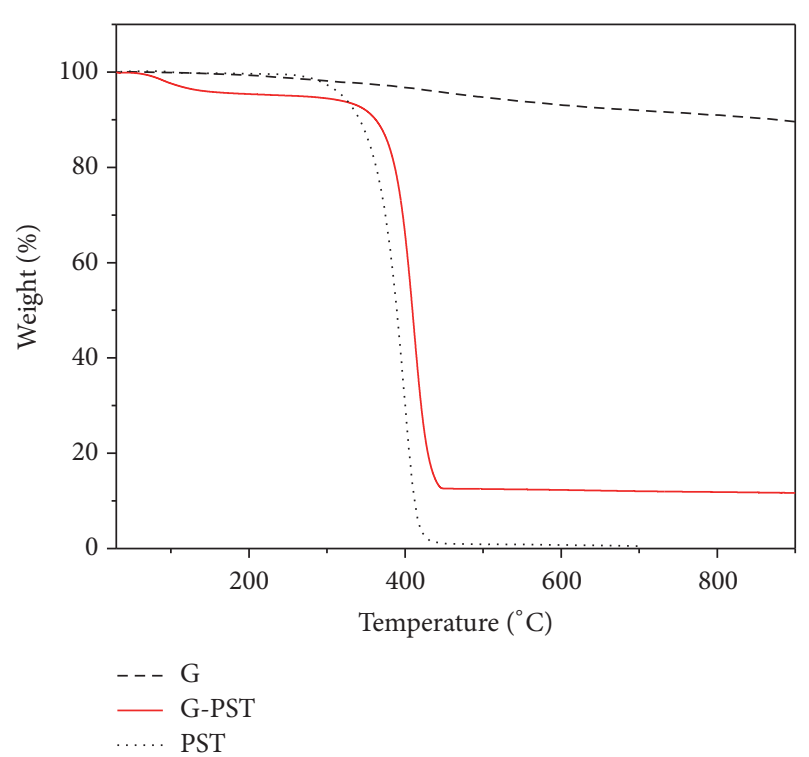

FIgUre 3: TGA analysis of the G and G-PST nanocomposite.

degradation process of pristine PST starts at around $305^{\circ} \mathrm{C}$ and ends at $410^{\circ} \mathrm{C}$.

The blank membrane was obtained according to Section 2.2.2 described previously. The phase inversion method used for the synthesis of polysulfone membranes drives the pores structure to asymmetric configuration, with small pores on the top and large pores at the bottom of the 


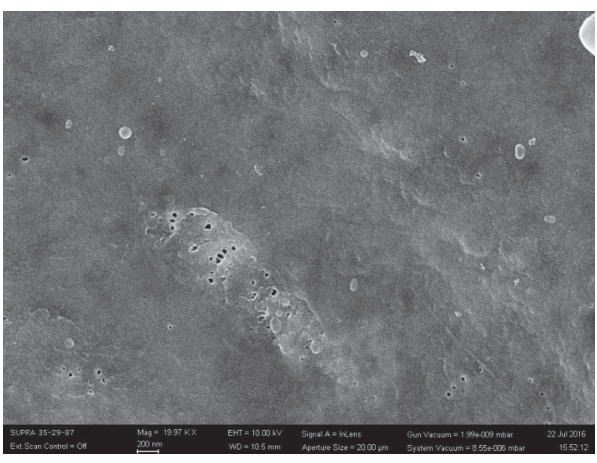

(a)

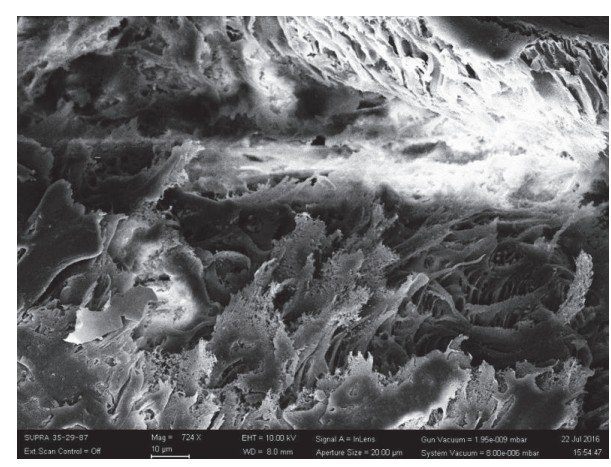

(b)

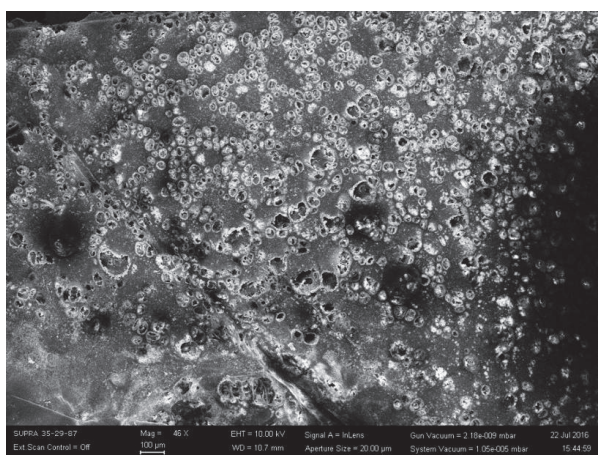

(c)

FIGURE 4: SEM image of polysulfone-graphene-polystyrene (PSF-G-PST) membrane: (a) top; (b) cross-section; (c) bottom.

membrane. According to Park et al. [29], at concentrations of $\mathrm{G}$ between 0.1 and $0.25 \%$ wt., the membranes cross-sections are characterized by finger-like pores which coexist with macrovoids at the bottom of the membrane. In our case, due to the presence of the G-PST composite the cross-section reveals finger-like pores close to the top and a sponge-like structure inside the membrane (Figure 4(b)). This could be explained by the presence of polystyrene, which is a more hydrophobic polymer that hinders the exchange between the solvent and the nonsolvent during coagulation time although the concentration of G-PST was kept to $0.2 \%$ wt.

Figure 4(a) confirms the formation of small pores (around $20-30 \mathrm{~nm}$ ), while the bottom of the membrane is characterized by large voids $(50-100 \mu \mathrm{m})$ with smaller pores aggregated inside their structure or independent small pores $(10-20 \mu \mathrm{m})$ formed in different areas of the membrane (Figure $4(\mathrm{c})$ ). This configuration confirms that the presence of the G-PST does not alter significantly the asymmetric structure of the final membrane at this loading concentration.

Aiming also at the enhancement of antimicrobial properties of our composite membranes, the next step of this study consisted in the electrochemical generation of $\mathrm{ZnO}$. In order to insert a high content of $\mathrm{ZnO}$ in the structure of the composite membrane, flat sheets were cut $(1 \times 1 \mathrm{~cm})$ and positioned with the bottom pores facing the opposite direction of the electrical field. This strategy allows the growth and aggregation of $\mathrm{ZnO}$ structures inside the larger pores. Filling the pores with $\mathrm{ZnO}$ will eventually create a compact structure that will favour a better contact between contaminated water and antimicrobial compound.
The electrochemical deposition of $\mathrm{ZnO}$ on the composite membrane intended also the attachment of the inorganic particles inside the macrovoids, deeper in the structure of the pores. However, the cross-section image (Figure 5(a)) presents the finger-like pores (close to the skin of the composite membrane) without $\mathrm{ZnO}$ particles inside the pores. The bottom configuration shows $\mathrm{ZnO}$ nanoparticles attached both on the surface and at the edges of the macrovoids (Figure 5(b)). The detailed images (Figures 5(c) and 5(d)) show on one hand the aggregates of $\mathrm{ZnO}$ concentrated on the edges of the larger pores and the attachment of inorganic particles (size less than $1 \mu \mathrm{m}$ ) on the walls of the interior pores on the other.

According to previous studies, the morphology of inorganic particles generated in electrical field can be severely influenced by the presence of water soluble polymers [20, 30]. In order to favour the formation of $\mathrm{ZnO}$ nanoparticles with controlled structure and narrow size distribution of the particles, the electrochemical deposition of $\mathrm{ZnO}$ on the PSFG-PST membrane was performed in the presence of water soluble polymers following the conditions from Table 1 .

In Figure 6, the presence of PVA in the electrochemical bath allowed the generation of $\mathrm{ZnO}$ structures with cubic shapes (associated with zinc blende crystalline structure of $\mathrm{ZnO}$ ) of different sizes ranging from 100 to $400 \mathrm{~nm}$. The bottom surface of the composite membrane reveals large inorganic structures of $\mathrm{ZnO}$ that were generated on the surface in different areas of the membrane (Figures 6(b) and 6(c)). The presence of PVA at this concentration was unable to inhibit the growth of $\mathrm{ZnO}$ particles making it difficult to drive 


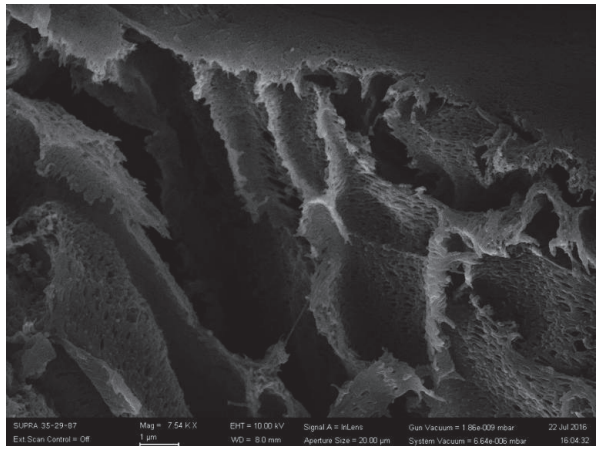

(a)

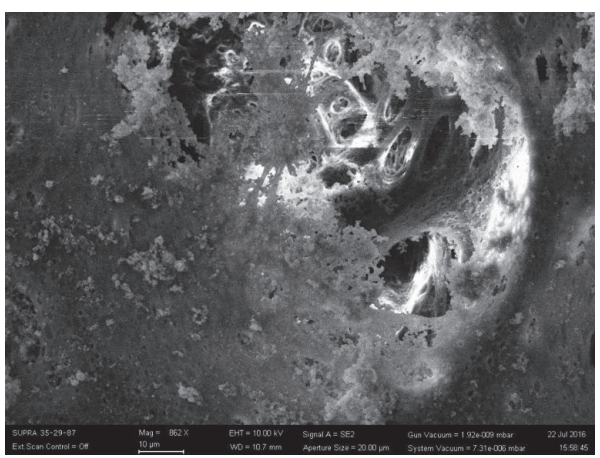

(c)

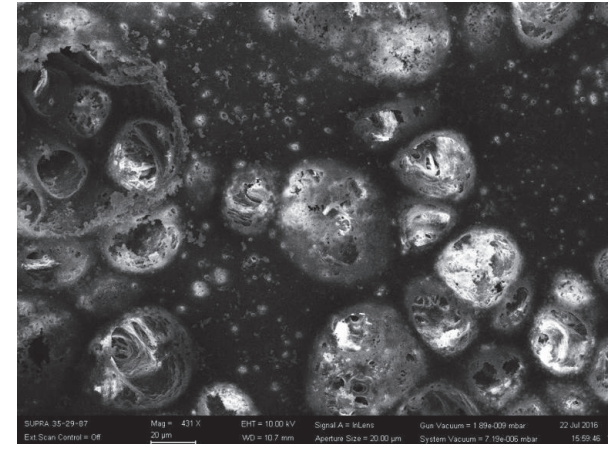

(b)

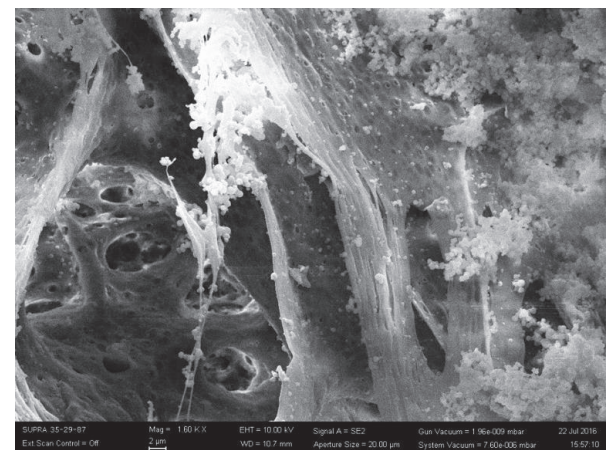

(d)

FIGURE 5: SEM images of PSF-G-PST-ZnO membrane: (a) cross-section; (b) bottom; (c, d) details of macrovoids from the bottom surface.

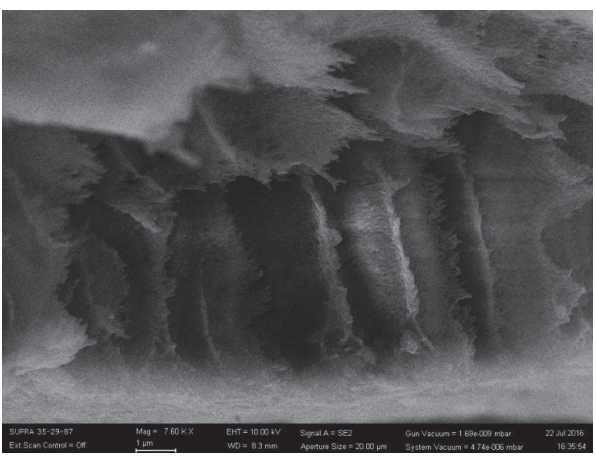

(a)

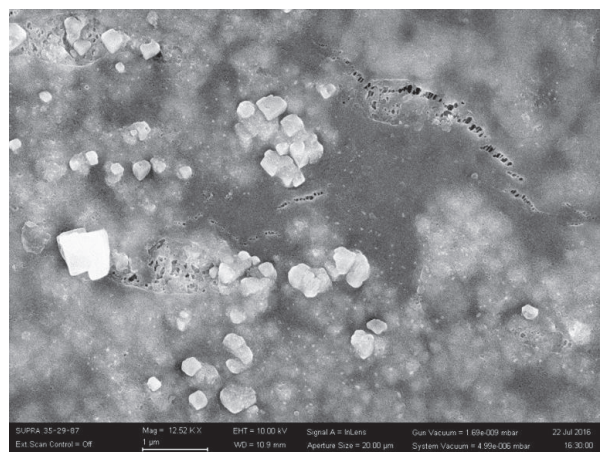

(b)

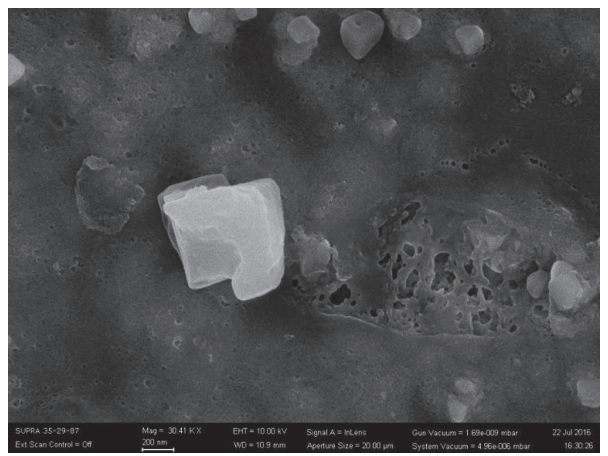

(c)

FIGURE 6: SEM images of ZnO deposition on PSF-G-PST in the presence of PVA (2.5\% wt.): (a) cross-section; (b) bottom; (c) detailed image of the bottom surface. 

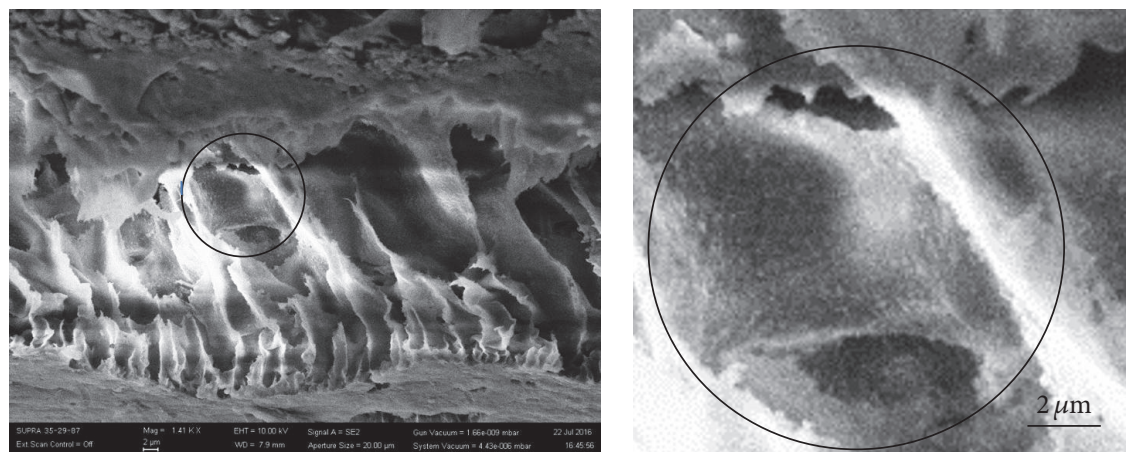

(a)

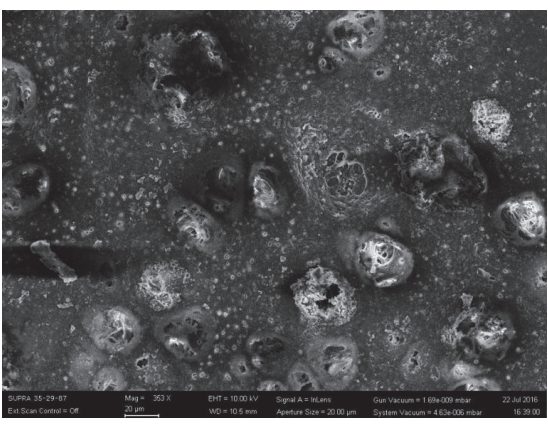

(b)

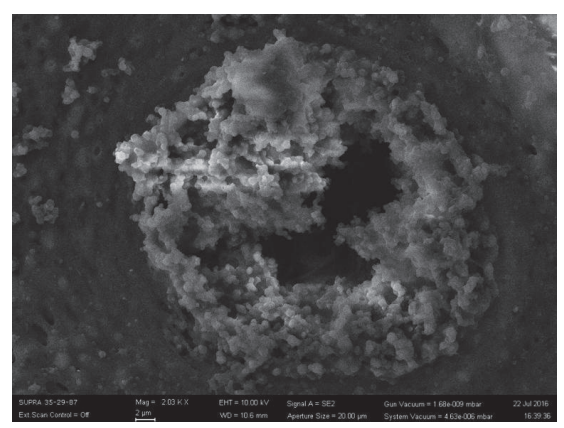

(c)

Figure 7: SEM analysis of ZnO deposited on PSF-G-PST membrane in the presence of PVA (10\% wt.): (a) cross-section; (b) bottom; (c) detailed image of the bottom surface.

the growth of the inorganic particles inside the macrovoids or the pore walls. Although spherical $\mathrm{ZnO}$ particles are present on the membrane (Figure 6(b)), the cross-section (Figure 6(a)) did not confirm the presence of such particles inside the structure that could have served as nucleation centers for the growth of the inorganic compound. The detailed image of the bottom surface presents the formation of small pores coexisting with larger pores (Figure 6(c)) that do not allow the generation of $\mathrm{ZnO}$ inside the structure.

For the next experiment, the generation of $\mathrm{ZnO}$ was conducted in the presence of higher concentration of PVA, knowing the fact that polymer solutions with higher viscosity will considerably reduce the size of the particles, affecting the crystal nucleation and growth process [31].

In the case of using $10 \%$ wt PVA the bottom surface of the composite membrane PSF-G-PST-PVA10 is characterized by large pores (around $20 \mu \mathrm{m})$ and small pores $(0.5-1 \mu \mathrm{m})$ (Figure 7(b)). The $\mathrm{ZnO}$ particles are drastically changed compared to the previous experiment. $\mathrm{ZnO}$ is concentrated on the whole surface of the back-side membrane and aggregated at the edge of the macrovoids. This can be attributed to the edges of the macrovoids which can be assimilated as defects into the composite material/deposition substrate for the nucleation and growth process of inorganic crystallites $[32,33]$. The detailed image (Figure $7(\mathrm{c})$ ) proves that the presence of higher concentration of PVA acts not only as an inhibitor, but also as a stabilizer of the inorganic particles; the spherical shape of $\mathrm{ZnO}$ particles is well distinguishable ranging from 0.5 to $1 \mu \mathrm{m}$ in size.

Furthermore, in Figure 7(a) (black circles) the inorganic $\mathrm{ZnO}$ particles were obtained on the pores walls for the first time in our experiments.

The presence of well-dispersed graphene inside the composite membrane assisted by the high voltage applied to the water soluble polymer solution, the size of the macrovoids, and the higher concentration of PVA allowed the formation of $\mathrm{ZnO}$ nucleus deeper into the membrane structure favouring the growth of inorganic spherical particles (with size in the range of $0.5-1 \mu \mathrm{m}$ ) on the porous walls.

Synthesis of inorganic particles in the presence of water soluble polymers is strongly influenced by the molecular weight of the polymer and also by the functional groups [3437].

Thus, in the next experiment, the $\mathrm{ZnO}$ nanostructures were generated in the presence of PAA at low concentration (2.5\% wt.)

Similar to PSF-G-PST-PVA 2.5 membrane, the crosssection did not show the presence of any zinc oxide particles inside the porous structure of the membrane. Thus, at this concentration, the amount of PAA inhibited the crystal growth process of the $\mathrm{ZnO}$ nanoparticles. Figures 8(b) and 8(c) exhibited individual or aggregated lamellar structures of $\mathrm{ZnO}$ that covered the bottom surface as a continuous layer for large areas, or aggregated at the edge of the macrovoids. The presence of certain functional groups (hydroxyl, carboxyl, 


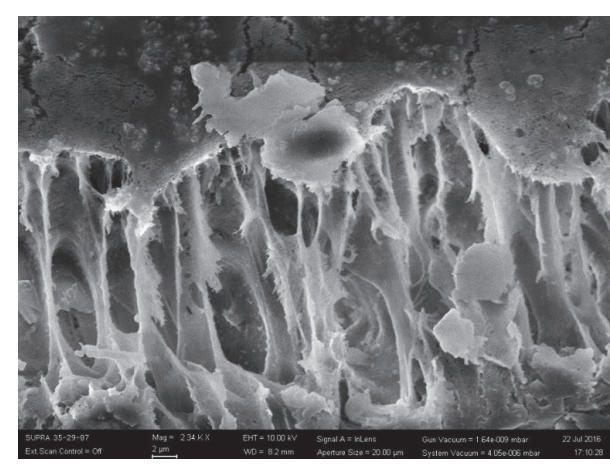

(a)

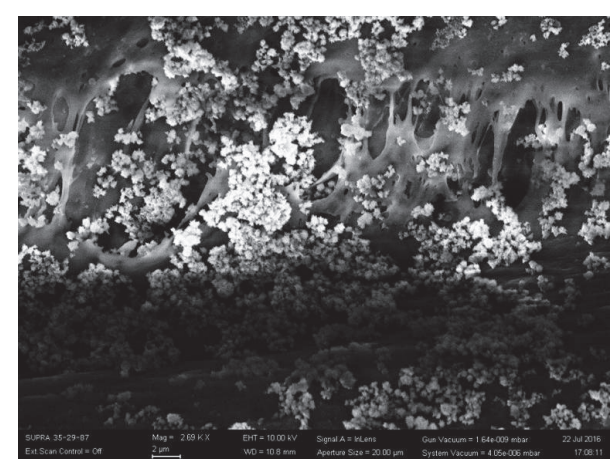

(b)

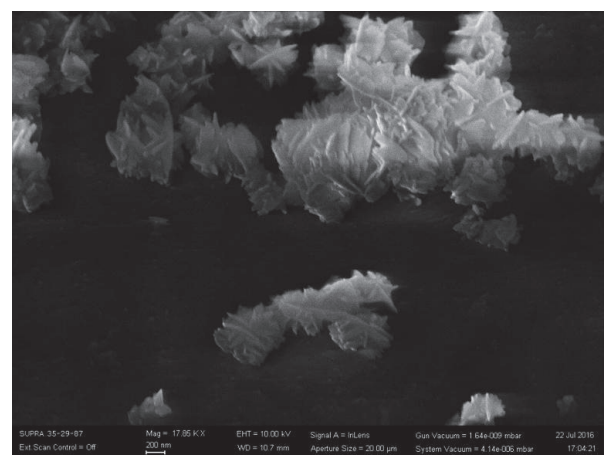

(c)

FIGURE 8: SEM analysis of ZnO deposited on PSF-G-PST membrane in the presence of PAA (2.5\% wt): (a) cross-section; (b) bottom; (c) detailed image of the bottom surface.

etc.) influences the configuration of $\mathrm{ZnO}$ nanostructures confirming that the functional groups of the water soluble compounds have a major influence to the formation of diverse shapes with different sizes [30].

Hydroxyethyl cellulose (HEC) is a water soluble polymer derived from cellulose and usually used as thickening agent in cosmetics, cleaning products, and drug or oilfield chemical products. Recent studies confirmed that HEC has an enhancing inhibitory effect in crystal growth processes in aliquots with poly(N-vinyl pyrrolidone) (PNVP) compared to polyethylene glycol (PEG) and polyacrylamide (PAM) [38]. The presence of HEC in $26 \% \mathrm{NH}_{4} \mathrm{Cl}$ solution, commonly used for zinc-carbon batteries, led to a decrease of the corrosion process which was attributed to a suppression effect of the anode and cathode reactions due to the adsorbed molecules of HEC that act as a protective layer for the zinc elements [39].

Based on these aspects, our experiment was carried out in the presence of HEC dissolved together with the $\mathrm{ZnO}$ precursors. After $1 \mathrm{~h}$ deposition time the samples were removed from the electrochemical bath and analysed by SEM (Figure 9).

The SEM analysis shows a clear evidence of the $\mathrm{ZnO}$ generated inside the structure of the pores (black circles marked in Figure 9(a)) in the case of the cross-section of the composite membrane. HEC was probably adsorbed on the first nucleus of the inorganic particles which were simultaneously attached to the porous walls embedded with graphene. Thus, HEC acted like a strong stabilizer that also inhibited the crystal growth of $\mathrm{ZnO}$ nanostructures.

Compared to PSF-G-PST-PVA10 membrane, where $\mathrm{ZnO}$ was also generated inside the porous structure (see Figure 7(a)), in this case the interior walls of the pores were covered with higher amounts of inorganic particles. Related to our previous experiments, the presence of HEC enhanced the deposition rate of $\mathrm{ZnO}$ inside the porous structure of the membrane, filling the macrovoids with spherical $\mathrm{ZnO}$ nanoparticles and covering the bottom surface on very large areas (Figure 9(b)). Figure 9(c) confirms that sphericalshaped $\mathrm{ZnO}$ was not only deposited on the edges of the bottom macrovoids, but also aggregated under electrical voltage filling-up the pores.

In conclusion, the use of HEC improved the deposition rate of $\mathrm{ZnO}$ nanostructures and favoured the electrochemical generation of the inorganic spheres in the whole structure of the composite membrane compared to the other water soluble polymer presented/used in this study.

In order to confirm the formation of $\mathrm{ZnO}$ on the membranes surface EDS analysis was performed on the bottom side (Figure 10).

The EDS spectrum demonstrates the presence of zinc $(\mathrm{Zn})$ and oxygen $(\mathrm{O})$ peaks together with a strong signal from carbon $(\mathrm{C})$ peak attributed to the composite membrane. Thus, this result validates the deposition of $\mathrm{ZnO}$ on the bottom surface of the hybrid membrane. 


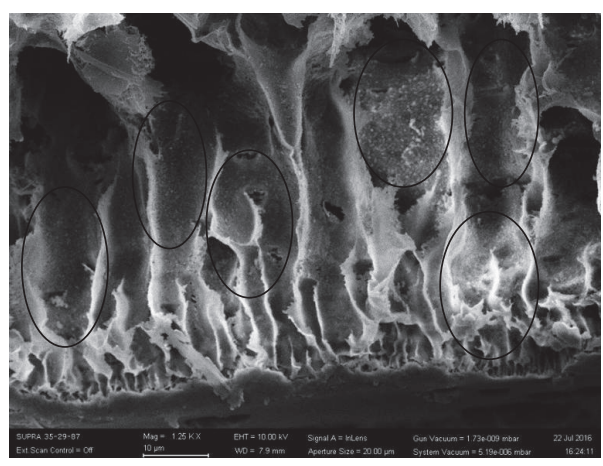

(a)

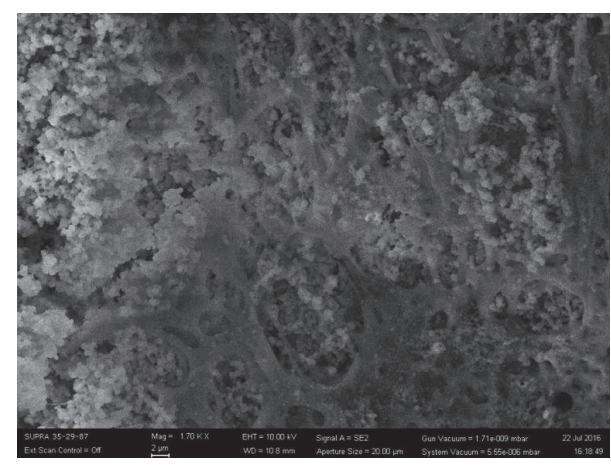

(b)

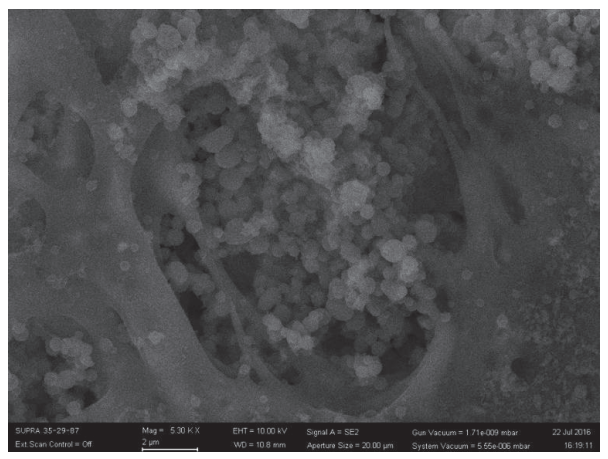

(c)

FIGURE 9: SEM image of ZnO deposited on PSF-G-PST in the presence of HEC: (a) cross-section; (b) bottom; (c) detailed image of the bottom surface.
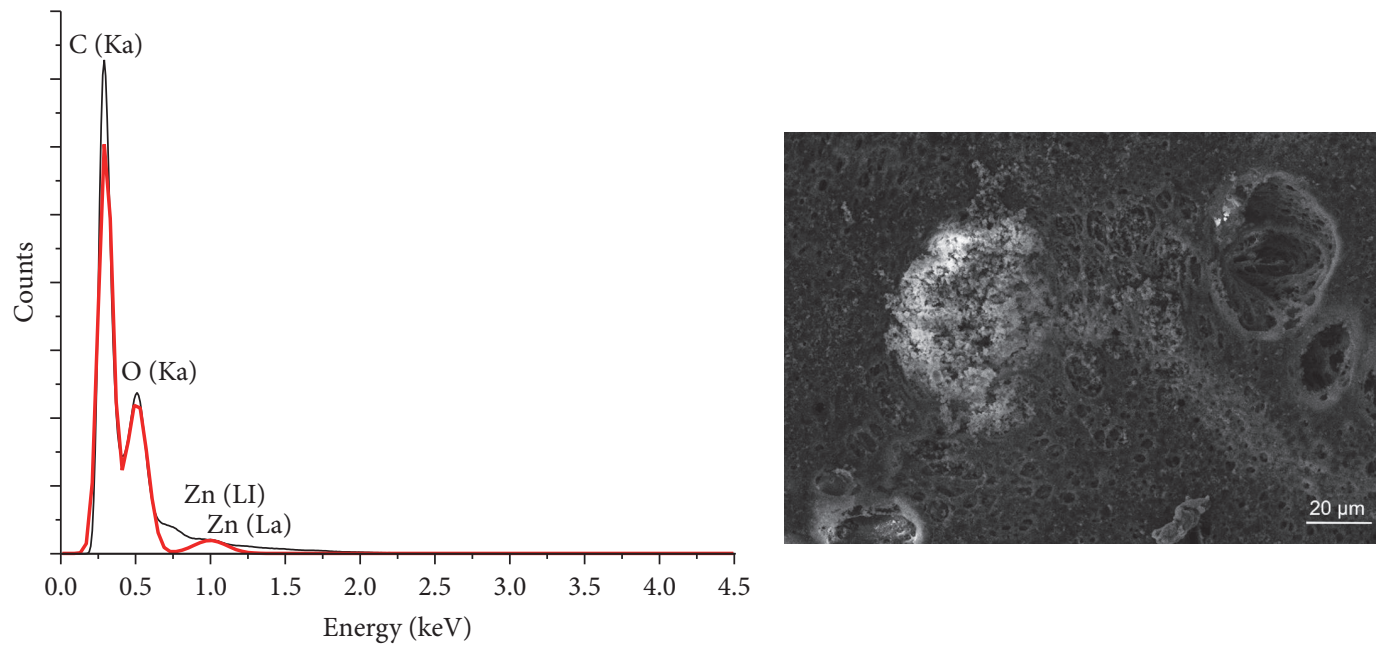

\begin{tabular}{lcc}
\hline Element & Weight \% & Atomic \% \\
\hline $\mathrm{C}(\mathrm{Ka})$ & 57.41 & 65.49 \\
$\mathrm{O}(\mathrm{Ka})$ & 39.55 & 33.87 \\
$\mathrm{Zn}(\mathrm{L})$ & 3.04 & 0.64 \\
\hline Total & 100 & 100 \\
\hline
\end{tabular}

FIGURE 10: SEM EDS and SEM analyses for ZnO deposited on the PSF-G-PST-membranes. 


\section{Conclusions}

The aim of this work was to develop an alternative synthesis procedure for hybrid polysulfone membranes endowed combined properties (i.e., mechanical and thermal resistance, antifouling and antimicrobial characteristics) in one single membrane designed for water purification.

In this work, graphene nanoplatelets were modified with PST in order to obtain polysulfone composite membranes relevant for electrochemical deposition of $\mathrm{ZnO}$. The deposition procedure was performed on one side of the composite membrane, at high voltage, in the presence of water soluble polymers.

At low concentrations $(2.5 \% \mathrm{wt})$ of water soluble polymers, such as PVA and PAA, the generation of $\mathrm{ZnO}$ nanostructures was influenced by the functional groups. In this case, the inorganic structures were generated mainly at the edges of the bottom macropores hardly protruding inside the pore channels.

The experiments confirmed that $\mathrm{ZnO}$ nanostructures can be embedded inside the porous structure of the composite membrane at high concentrations of PVA (around 10\% wt.).

The use of water soluble polymers with strong inhibitory effect on the crystal growth process, such as HEC, improved the deposition rate, decreased the size of inorganic structures, and increased the amount of $\mathrm{ZnO}$ deposited in the whole structure of the composite PSF-G-PST-membrane. These results are promising and further research will be performed to reach the goal of obtaining new composite materials for improved efficiency membranes for water purification systems.

\section{Conflicts of Interest}

The authors declare that there are no conflicts of interest regarding the publication of this paper.

\section{Acknowledgments}

The authors would like to acknowledge the financial support provided by the National Authority for Scientific Research from the Ministry of Education, Research and Youth of Romania through the PN-II-PT-PCCA-2013-4-0203-NITROMIP Project.

\section{References}

[1] S. Alzahrani and A. W. Mohammad, "Challenges and trends in membrane technology implementation for produced water treatment: a review," Journal of Water Process Engineering, vol. 4, pp. 107-133, 2014.

[2] J. Hong and Y. He, "Effects of nano sized zinc oxide on the performance of PVDF microfiltration membranes," Desalination, vol. 302, pp. 71-79, 2012.

[3] S. R. Panda and S. De, "Preparation, characterization and performance of $\mathrm{ZnCl}_{2}$ incorporated polysulfone (PSF)/polyethylene glycol (PEG) blend low pressure nanofiltration membranes," Desalination, vol. 347, pp. 52-65, 2014.

[4] N. A. A. Hamid, A. F. Ismail, T. Matsuura et al., "Morphological and separation performance study of polysulfone/titanium dioxide $\left(\mathrm{PSF} / \mathrm{TiO}_{2}\right)$ ultrafiltration membranes for humic acid removal," Desalination, vol. 273, no. 1, pp. 85-92, 2011.

[5] M. R. Esfahani, J. L. Tyler, H. A. Stretz, and M. J. M. Wells, "Effects of a dual nanofiller, nano- $\mathrm{TiO}_{2}$ and MWCNT, for polysulfone-based nanocomposite membranes for water purification," Desalination, vol. 372, pp. 47-56, 2015.

[6] Q. Ma, H. Zhang, J. Zhao, and Y.-K. Gong, "Fabrication of cell outer membrane mimetic polymer brush on polysulfone surface via RAFT technique," Applied Surface Science, vol. 258, no. 24, pp. 9711-9717, 2012.

[7] H. Rabiee, V. Vatanpour, M. H. D. A. Farahani, and H. Zarrabi, "Improvement in flux and antifouling properties of PVC ultrafiltration membranes by incorporation of zinc oxide $(\mathrm{ZnO})$ nanoparticles," Separation and Purification Technology, vol. 156, part 2, pp. 299-310, 2015.

[8] R. Mukherjee and S. De, "Novel carbon-nanoparticle polysulfone hollow fiber mixed matrix ultrafiltration membrane: adsorptive removal of benzene, phenol and toluene from aqueous solution," Separation and Purification Technology, vol. 157, pp. 229-240, 2016.

[9] D. Y. Koseoglu-Imer, B. Kose, M. Altinbas, and I. Koyuncu, "The production of polysulfone (PS) membrane with silver nanoparticles (AgNP): physical properties, filtration performances, and biofouling resistances of membranes," Journal of Membrane Science, vol. 428, pp. 620-628, 2013.

[10] S. Li, M. Meng Lin, M. Toprak, D. K. Kim, and M. Muhammed, "Nanocomposites of polymer and inorganic nanoparticles for optical and magnetic applications," Nano Reviews, vol. 1, no. 1, article 5214, 2017.

[11] G. Schmidt and M. M. Malwitz, "Properties of polymernanoparticle composites," Current Opinion in Colloid and Interface Science, vol. 8, no. 1-2, pp. 103-108, 2003.

[12] L. Dai, D. W. Chang, J.-B. Baek, and W. Lu, "Carbon nanomaterials for advanced energy conversion and storage," Small, vol. 8, no. 8, pp. 1130-1166, 2012.

[13] Z. Spitalsky, D. Tasis, K. Papagelis, and C. Galiotis, "Carbon nanotube-polymer composites: chemistry, processing, mechanical and electrical properties," Progress in Polymer Science, vol. 35, no. 3, pp. 357-401, 2010.

[14] Z. Wang, H. Yu, J. Xia et al., "Novel GO-blended PVDF ultrafiltration membranes," Desalination, vol. 299, pp. 50-54, 2012.

[15] N. Meng, R. C. E. Priestley, Y. Zhang, H. Wang, and X. Zhang, "The effect of reduction degree of GO nanosheets on microstructure and performance of PVDF/GO hybrid membranes," Journal of Membrane Science, vol. 501, pp. 169-178, 2016.

[16] E. Rusen, A. Mocanu, L. C. Nistor et al., "Design of antimicrobial membrane based on polymer colloids/multiwall carbon nanotubes hybrid material with silver nanoparticles," ACS Applied Materials and Interfaces, vol. 6, no. 20, pp. 17384-17393, 2014.

[17] G. Jung and H.-I. Kim, "Synthesis and photocatalytic performance of PVA/TiO $/$ /graphene-MWCNT nanocomposites for dye removal," Journal of Applied Polymer Science, vol. 131, no. 17, pp. 8797-8803, 2014.

[18] E. Mahmoudi, L. Y. Ng, M. M. Ba-Abbad, and A. W. Mohammad, "Novel nanohybrid polysulfone membrane embedded with silver nanoparticles on graphene oxide nanoplates," Chemical Engineering Journal, vol. 277, pp. 1-10, 2015.

[19] V. Kaiser, C. Stropnik, V. Musil, and M. Brumen, "Morphology of solidified polysulfone structures obtained by wet phase separation," European Polymer Journal, vol. 43, no. 6, pp. 25152524, 2007. 
[20] A. Mocanu, E. Rusen, A. Diacon, and A. Dinescu, "Hierarchical nanostructures of $\mathrm{PbS}$ obtained in the presence of water soluble polymers," Powder Technology, vol. 253, pp. 237-241, 2013.

[21] S. Stankovich, D. A. Dikin, R. D. Piner et al., "Synthesis of graphene-based nanosheets via chemical reduction of exfoliated graphite oxide," Carbon, vol. 45, no. 7, pp. 1558-1565, 2007.

[22] H. Wang, Y. Wang, X. Cao, M. Feng, and G. Lan, "Vibrational properties of graphene and graphene layers," Journal of Raman Spectroscopy, vol. 40, no. 12, pp. 1791-1796, 2009.

[23] F. M. Koehler, A. Jacobsen, T. Ihn, K. Ensslin, and W. J. Stark, "Chemical modification of graphene characterized by Raman and transport experiments," Nanoscale, vol. 4, no. 12, pp. 37813785, 2012.

[24] E. Paparazzo, "On the interpretation of XPS spectra of metal (Pt, Pt-Sn) nanoparticle/graphene systems," Carbon, vol. 63, pp. 578-581, 2013.

[25] N. Cai and P. Larese-Casanova, "Application of positivelycharged ethylenediamine-functionalized graphene for the sorption of anionic organic contaminants from water," Journal of Environmental Chemical Engineering, vol. 4, no. 3, pp. 29412951, 2016.

[26] B. Xue, J. Zhu, N. Liu, and Y. Li, "Facile functionalization of graphene oxide with ethylenediamine as a solid base catalyst for Knoevenagel condensation reaction," Catalysis Communications, vol. 64, pp. 105-109, 2015.

[27] Z. Zhao, J. Li, T. Wen, C. Shen, X. Wang, and A. Xu, "Surface functionalization graphene oxide by polydopamine for high affinity of radionuclides," Colloids and Surfaces A: Physicochemical and Engineering Aspects, vol. 482, pp. 258-266, 2015.

[28] M.-S. Park and Y.-S. Lee, "Functionalization of graphene oxide by fluorination and its characteristics," Journal of Fluorine Chemistry, vol. 182, pp. 91-97, 2016.

[29] M. J. Park, S. Phuntsho, T. He et al., "Graphene oxide incorporated polysulfone substrate for the fabrication of flat-sheet thin-film composite forward osmosis membranes," Journal of Membrane Science, vol. 493, pp. 496-507, 2015.

[30] F. Miculescu, E. Rusen, A. Mocanu, A. Diacon, and R. Birjega, "Hierarchical nanostructures of $\mathrm{ZnO}$ obtained in the presence of water soluble polymers," Powder Technology, vol. 239, pp. 5658, 2013.

[31] S. Ozaki, I. Kushida, T. Yamashita, T. Hasebe, O. Shirai, and K. Kano, "Inhibition of crystal nucleation and growth by watersoluble polymers and its impact on the supersaturation profiles of amorphous drugs," Journal of Pharmaceutical Sciences, vol. 102, no. 7, pp. 2273-2281, 2013.

[32] B. K. Min, W. T. Wallace, A. K. Santra, and D. W. Goodman, "Role of defects in the nucleation and growth of Au nanoclusters on $\mathrm{SiO}_{2}$ thin films," The Journal of Physical Chemistry B, vol. 108, no. 42, pp. 16339-16343, 2004.

[33] R. Conrad and M. Harsdorff, "Influence of surface defects on the nucleation and growth of thin discontinuous gold films," International Journal of Electronics, vol. 69, no. 1, pp. 153-167, 1990.

[34] P. Pramanik, "Synthesis of nano particle of inorganic oxides by polymer matrix," Bulletin of Materials Science, vol. 18, no. 6, pp. 819-829, 1995.

[35] H. Wei, Q. Shen, Y. Zhao, D.-J. Wang, and D.-F. Xu, "Influence of polyvinylpyrrolidone on the precipitation of calcium carbonate and on the transformation of vaterite to calcite," Journal of Crystal Growth, vol. 250, no. 3-4, pp. 516-524, 2003.
[36] C. Wang, T. Shen, S. Li, and X. Wang, "Investigation of influence of low phosphorous co-polymer antiscalant on calcium sulfate dihydrate crystal morphologies," Desalination, vol. 348, pp. 8993, 2014.

[37] D. Peronno, H. Cheap-Charpentier, O. Horner, and H. Perrot, "Study of the inhibition effect of two polymers on calcium carbonate formation by fast controlled precipitation method and quartz crystal microbalance," Journal of Water Process Engineering, vol. 7, pp. 11-20, 2015.

[38] E. F. Jokandan, P. Naeiji, and F. Varaminian, "The synergism of the binary and ternary solutions of polyethylene glycol, polyacrylamide and Hydroxyethyl cellulose to methane hydrate kinetic inhibitor," Journal of Natural Gas Science and Engineering, vol. 29, pp. 15-20, 2016.

[39] M. A. Deyab, "Hydroxyethyl cellulose as efficient organic inhibitor of zinc-carbon battery corrosion in ammonium chloride solution: electrochemical and surface morphology studies," Journal of Power Sources, vol. 280, pp. 190-194, 2015. 

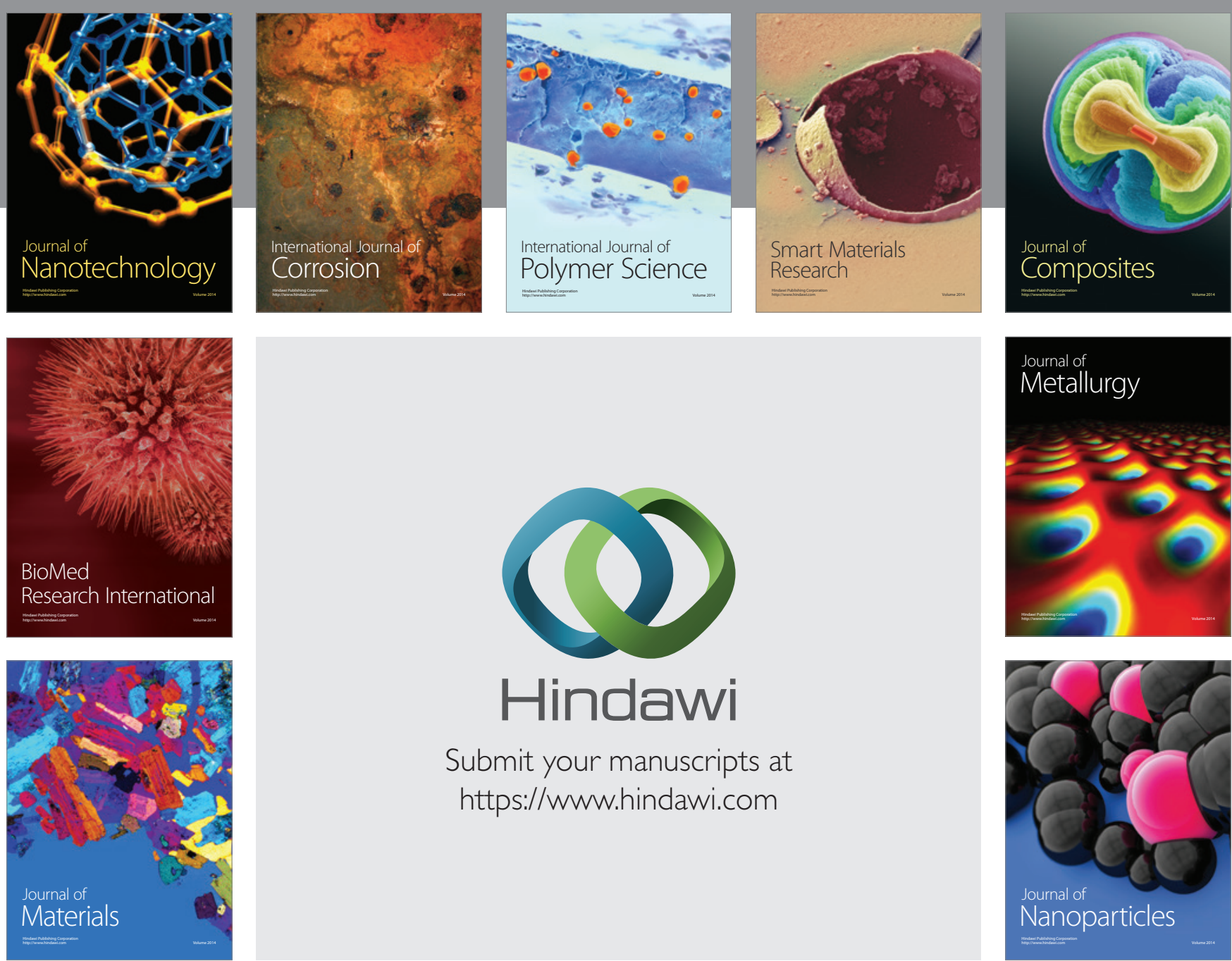

\section{Hindawi}

Submit your manuscripts at

https://www.hindawi.com

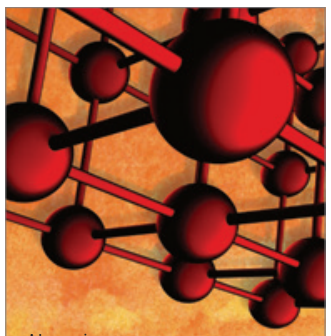

Materials Science and Engineering
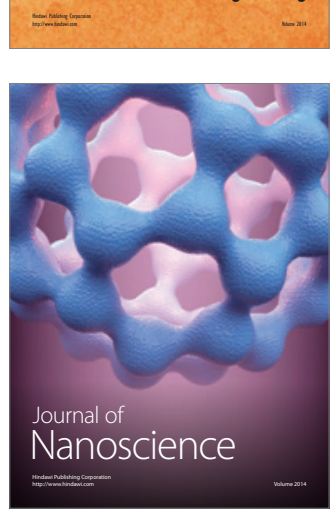
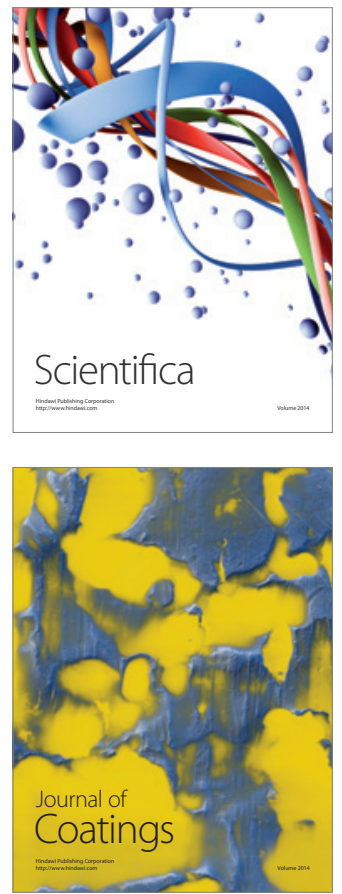
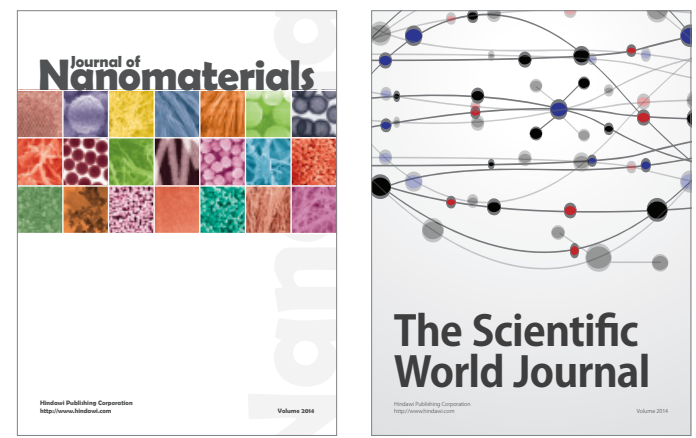

The Scientific World Journal
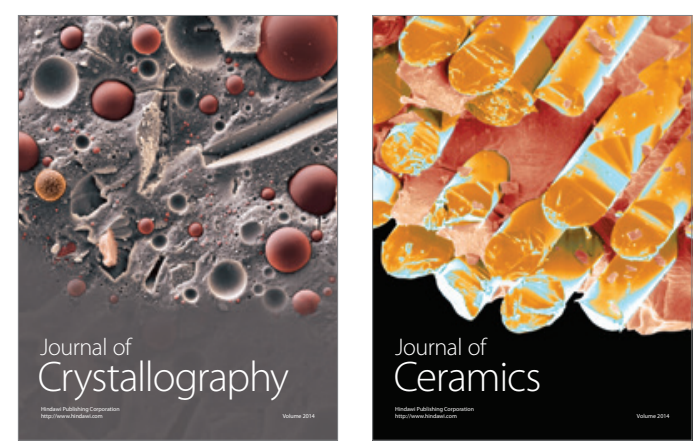
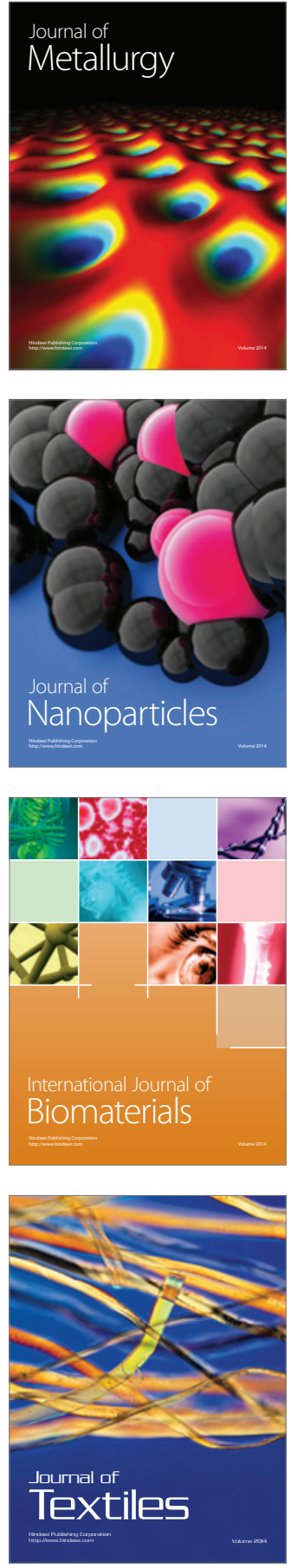